\title{
Aplicação da Avaliação do Ciclo de Vida na análise de impactos ambientais de materiais de construção inovadores: estudo de caso da pegada de carbono de clínqueres alternativos
}

\author{
Application of Life Cycle Assessment in the evaluation of \\ the environmental impacts of innovative construction \\ materials: case study of the carbon footprint of \\ alternative clinkers
}

\section{Ana Carolina Badalotti Passuello \\ Alexandre Führ de Oliveira \\ Eugênio Bastos da Costa \\ Ana Paula Kirchheim}

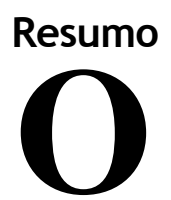

setor da construção civil é reconhecido como um grande consumidor de recursos, e responsável por diversas emissões que causam impactos substanciais ao ambiente. Muitos destes impactos estão diretamente relacionados à produção de materiais de construção. Por isso, mundialmente cresce a tendência de se propor o uso de materiais de construção inovadores, que considerem a mitigação de seus impactos ambientais no processo produtivo. Para avaliar todos os impactos ambientais relacionados a estes materiais, é necessário que se avalie todas as fases de seu ciclo de vida. Para isso, propõe-se a aplicação da Avaliação do Ciclo de Vida (ACV), metodologia internacionalmente consagrada na avaliação ambiental de produtos, processos e serviços, sendo também normatizada para o cálculo de declarações ambientais de produtos da construção civil. Sabe-se que a produção cimento emite quantidade pronunciadas de gases do efeito estufa. Por isso, neste trabalho, a ACV é aplicada para o cálculo da pegada de carbono de clínqueres alternativos produzidos em laboratório. Os resultados obtidos demonstram uma redução em $22 \%$ da pegada de carbono do clínquer alternativo em relação ao clínquer Portland, indicando as etapas do processo produtivo que mais colaboram para estes valores. Ainda, evidencia-se a ampla aplicabilidade da ferramenta ACV ao setor da construção.

Ana Carolina Badalotti Passuello Universidade Federal do Rio Grande Porto Alegre - RS - Brasil

Alexandre Führ de Oliveira Universidade Federal do Rio Grande do Sul Porto Alegre - RS - Brasil

Eugênio Bastos da Costa Universidade Federal do Rio Grande do Sul Porto Alegre - RS - Brasil

Ana Paula Kirchheim Universidade Federal do Rio Grande

Porto Alegre - RS - Brasil

Recebido em 15/03/14

Aceito em 11/09/14
Palavras-chaves: Avaliação do Ciclo de Vida. Pegada de Carbono. Clínquer alternativo. Clínquer Portland.

\section{Abstract}

It is widely acknowledged that the construction industry is a majorconsumer of resources and is responsible for emissions that cause substantial environmental impacts. Many of those impacts are directly related to the production of building materials. Consequently, the use of innovative building materials that consider the mitigation of environmental impacts through their production process has become a worldwide trend. In order to evaluate all the environmental impacts related to these materials, it is necessary to assess allthe life cycle stages. In order to do that, we propose the application of Life Cycle Assessment (LCA), an internationally acknowledged method for the environmental evaluation of products, processes and services, which is also standardized for the environmental product declaration of construction products. Cement production is known to emit significant amounts of greenhouse gases. Hence, in this paper, LCA is applied to evaluate the carbon footprint of alternative, laboratory-produced clinker. The results show a $22 \%$ reduction in the carbon footprint of alternative clinker when compared to Portland clinker, indicating the stages in the production process that most contribute to these values. Moreover, the results indicatea wide range of applications of LCA in the construction industry.

Keywords: Life-Cycle Assessment. Carbon Footprint. Alternative clinker. Portland clinker. 


\section{Introdução}

A construção civil é mundialmente reconhecida como um dos principais agentes causadores de contaminação ambiental na atualidade. Dados bibliográficos demonstram a importância do setor construtivo na pegada ecológica e na economia de um país. $\mathrm{Na}$ economia norte-americana, por exemplo, edificações residenciais representam $5,3 \%$ do PIB, $38 \%$ do consumo de eletricidade, $26 \%$ do consumo energético, $24 \%$ da emissão de gases efeito estufa, $26 \%$ da geração de resíduos perigosos e $12 \%$ da emissão de gases tóxicos (OCHOA; HENDRICKSON; MATTHEWS, 2002). Uma estimativa dos consumos de recursos, eletricidade e energia, emissões de gases do efeito estufa, resíduos perigosos gerados e emissões atmosféricas tóxicas de uma residência norteamericana típica demonstra que a fase de construção representa $46 \%$ da atividade econômica relacionada à edificação, sendo responsável por $57 \%$ das emissões atmosféricas registradas e contribuindo em $51 \%$ na geração de resíduos perigosos (OCHOA; HENDRICKSON; MATTHEWS,2002). Esses dados salientam a pronunciada relevância do setor na economia e gestão ambiental de um país desenvolvido, e valores similares são esperados em países em vias de desenvolvimento como o Brasil, pelo elevado crescimento do setor nos últimos anos.

O Brasil vem experimentando uma crescente demanda habitacional, fruto do proeminente desenvolvimento econômico do país e do consequente aumento do poder aquisitivo da população. Essa crescente demanda originou sérios impactos ao meio ambiente, devido a fatores como emissões associadas à produção de insumos, consumo energético devido à produção de materiais de construção e no canteiro de obras e crescente produção de resíduos da construção.

Tendo em conta que boa parte dos impactos econômicos e ambientais que podem ser evitados estáassociada à fase de construção, o desenvolvimento de novas tecnologias construtivas é essencial para minimizar os impactos desse setor. Além disso, a aplicação de análises preditivas da avaliação do impacto, do uso da energia e emissões de poluentes durante o ciclo de vida desses novos materiais permite que se produzam materiais ecoeficientes, para atender à demanda de um mercado cada vez mais exigente em matéria ambiental. Uma melhor gestão dos materiais de construção implica uma grande melhoria na pegada ecológica e de carbono da construção civil e na redução global de consumo de recursos naturais e de impactos associados à produção desses produtos.
Nesse sentido, a metodologia ACV realiza um balanço de todas as fases do ciclo de vida do produto em estudo - seja ele um insumo ou a edificação como um todo - e suas interações, que podem ocorrer no tempo e no espaço, e seus impactos relacionados. Ciclo de vida é a expressão usada para referir-se a todas as etapas e processos de um sistema de produtos ou serviços, englobando toda a cadeia de produção e consumo, considerando aquisição de energia, matériasprimas e produtos auxiliares; aspectos dos sistemas de transportes e logística; características da utilização, manuseio, embalagem e consumo; sobras e resíduos; e sua respectiva reciclagem ou destino final.

A aplicação dessa metodologia ao setor da construção civil é internacionalmente reconhecida, sendo previamente aplicada para o cálculo do ciclo de vida de materiais de construção como o cimento (HUNTZINGER; $\quad$ EATMON, 2009; VALDERRAMA et al.,2011), blocos cerâmicos (KORONEOS; DOMPROS, 2007), aberturas (NORRIS; YOST, 2001; ABEYSUNDRA et al., 2007), pavimentos (BISWAS, 2013; JÖNSSON; TILLMANN; SVENSSON, 1997; NICOLETTI; MOTARNICOLA; TASSIELLI, 2002; NEBEL; ZIMMER; WEGENER, 2006), materiais isolantes (HUIJBREGTS et al., 2003) e estruturas em bambu (LUGT et al., 2006). Porém, os impactos relativos ao ciclo de vida de produtos e processos estão altamente relacionados a características locais do estudo. Existe carência de estudos de ACV de materiais de construção no Brasil. Assim sendo, fazem-se necessários estudos que relacionem a prática da $\mathrm{ACV}$ ao cenário local, de modo a torná-la um instrumento de gestão aplicável à realidade brasileira.

Entre os materiais de construção tradicionalmente utilizados, destaca-se a ampla aplicabilidade do cimento Portland, cuja indústria é reconhecida como uma das principais fontes emissoras de gases do efeito estufa (GEE) na atualidade, sendo responsável pela emissão de aproximadamente $7 \%$ do somatório de todas as atividades antropogênicas (MEHTA; MONTEIRO, 2008). Bosoaga, Masek e Oakey(2009)reportam que essas emissões estão associadas a queima de combustíveis (aproximadamente 40\%), calcinação $(\sim 50 \%)$ e moagem e transporte $(\sim 10 \%)$. A produção do clínquer está diretamente relacionada a todas essas etapas. O último relatório do IPCC (INTERGOVERNMENTAL..., 2014) salienta a importância de medidas mitigatórias para reduzir as emissões de GEE relacionadas a essa indústria. Entre as principais alternativas, cabe destacar

8 Passuello, A. C. B.; Oliveira, A. F. de; Costa, E. B. da; Kirchheim, A. P. 
melhoras no processo produtivo e redução do fator clínquer, ou até mesmo o desenvolvimento de cimentos alternativos de baixo impacto ambiental. Nesse sentido, mundialmente estão sendo desenvolvidos diversos ligantes que aplicam resíduos em sua produção e que possuem emissões de GEE mais baixas em seu processo produtivo quando comparados aos cimentos Portland tradicionais. Desses ligantes, cabe destacar os cimentos sulfoaluminato de cálcio (CSA), os cimentos sulfoaluminato de cálcio belíticos (CSAB), cimentos álcali-ativados, entre outros.

A demanda por um mercado de uso de carbono consciente representa o principal motor para a adoção e a viabilização no curto prazo de cimentos alternativos.No entanto, existem lacunas quanto ao real benefício ambiental desses cimentos quando comparados ao Portland. Dessa forma, o objetivo deste trabalho é avaliar a pegada de carbono de clínqueres alternativos produzidos em laboratório. Para tanto, primeiramente é descrita a metodologia de ACV, com ênfase em seu emprego para a avaliação de materiais de construção. A seguir, descreve-se a aplicação da ferramenta ao estudo de caso selecionado, demonstrando a aplicabilidade da ferramenta por meio de um exemplo didático. Finalmente, são discutidos os resultados obtidos, as vantagens e as limitações da aplicação de ferramenta.

\section{Avaliação do Ciclo de Vida (ACV) e sua aplicação a materiais de construção}

A ACV é uma ferramenta usada para avaliar os impactos ambientais de produtos, processos ou serviços. É uma ferramenta "do berço ao túmulo" que pode ser usada para avaliar sistemas complexos como edificações. Essa metodologia está indicada pelo Conselho Nacional de Metrologia, Normalização e Qualidade Industrial (Conmetro) como base para a identificação de oportunidades para a melhoria do desempenho ambiental de produtos em diversos pontos de seu ciclo de vida, capaz de incrementar o nível de informação dos tomadores de decisão na indústria e nas organizações governamentais e nãogovernamentais (BRASIL, 2011). Por ser uma metodologia internacionalmente reconhecida na melhoria da sustentabilidade de processos e produtos, a aplicação da $\mathrm{ACV}$ na indústria brasileira promoverá o acesso aos mercados interno e externo, satisfazendo a expectativa de consumidores cada vez mais exigentes em relação ao perfil ambiental dos produtos adquiridos. A aplicação da ACV permite o cálculo do impacto da cadeia produtiva ao meio ambiente e o expressa através de distintas métricas (categorias de impacto), como, por exemplo, os impactos arecursos energéticos não renováveis (em MJ) e mudanças climáticas (carbonfootprint ou pegada de carbono, em $\mathrm{kg} \mathrm{CO}_{2} \mathrm{eq}$ ).

De acordo com a norma BRE 15804 (BUILDING..., 2013), que avalia o desempenho ambiental de produtos relacionados à indústria da construção civil, através da rotulagem ambiental EPD (do inglês Environmental ProductDeclaration, ou declarações ambientais de produtos), no ciclo de vida das edificações podemse identificar cinco fases:

(a) seleção de produtos;

(b) construção;

(c) uso;

(d) fim de vida; $\mathrm{e}$

(e) benefícios e cargas ambientais.

De acordo com essa norma, a etapa de seleção de produtos relativa à $\mathrm{ACV}$ dos materiais de construção aplicados na edificação é mandatória para todos os tipos de EPD, salientando-se a importância do projeto de edificações e da seleção dos materiais de construção ambientalmente comprometidos no impacto ambiental da edificação como um todo. Essa etapa compreende o suprimento de matérias-primas, transporte e manufatura desses produtos. Já a etapa de construção aborda o transporte de materiais ao canteiro de obras e a construção propriamente dita. A fase de uso compreende a operação da edificação (uso de água e energia) e todas as fases de manutenção e reparo. A fase de fim de vida considera a demolição da edificação, o transporte de resíduos, seu processamento e disposição. Finalmente, a etapa de benefícios e cargas ambientais além das fronteiras do sistema de estudo considera o potencial de reúso, recuperação e reciclagem de resíduos produzidos ao longo do ciclo devida $(\mathrm{CV})$.

É consenso nas publicações relacionadas à $\mathrm{ACV}$ de edificações que a fase de uso seja a que possui maior consumo energético (ORTIZ; CASTELL; SONNEMANN, 2009), a qual contribui significativamente para sua pegada de carbono. Porém, como existe uma tendência de redução do consumo energético pela melhor eficiência da edificação, além da disponibilidade de eletrodomésticos e lâmpadas de baixo consumo, estima-se que esta fase tenha sua importância reduzida com o passar dos anos, e que outras fases, tais como a seleção de materiais de construção, tornem-se ainda mais importantes (PASSER; KREINER; MAYDL, 2012). 
Com base na série NBR/ISO 14040 (ABNT, 2009), a ACV tem quatro etapas:

(a) definição de objetivo e escopo;

(b) análise de inventário (ICV);

(c) avaliação de impactos (AICV); e

(d) interpretação.

O escopo define os meios para atingir os objetivos do estudo. Nesta etapa definem-se a fronteira do sistema ou a abrangência do estudo, especificando quais processos elementares fazem parte do sistema de produto. Também é definido o grau de detalhamento, de acordo com o objetivo e o uso pretendido para o estudo. $\mathrm{Na}$ fase de ICV é realizado um inventário de dados de entradas e saídas associados ao sistema de estudo. Já a fase de AICV provê informações adicionais a respeito dos impactos associados ao produto/processo em estudo, ampliando o entendimento de sua significância ambiental. Finalmente, na interpretação, os resultados são sumarizados e discutidos, fornecendo base para conclusões, recomendações e tomada de decisão, de acordo com a definição do objetivo e escopo. A seguir, são comentadas algumas especificidades da aplicação da ACV a materiais de construção, com base nas descrições das normas em vigor (ABNT, 2009; BUILDING..., 2013).

\section{Definição de objetivo e escopo}

O objetivo do estudo deve conter a aplicação pretendida, as razões para a realização do estudo, o público-alvo e referência à intenção de utilizar os resultados em afirmações comparativas a serem divulgadas publicamente (ABNT, 2009). Já o escopo define uma série de itens necessários para a realização do estudo, tais como o sistema de produto e suas funções, a fronteira do sistema, a unidade funcional, procedimentos de alocação, metodologia de AICV e impactos a serem avaliados, requisitos de qualidade dos dados, entre outros (ABNT, 2009).

A unidade funcional é uma quantidade definida do produto em estudo, usada como referência para calcular seu desempenho. Essa unidade permite que diferentes sistemas de produto com a mesma função possam ser comparados. No caso da ACV relacionada à construção civil, para o cálculo de declarações ambientais de produto, a unidade funcional deve ser baseada (BUILDING..., 2013):

(a) no uso relevante quantificado ou no desempenho característico do produto quando aplicado à construção; e

(b) na vida útil de serviço do produto, ou na vida útil da edificação, por relacionar o desempenho funcional do produto ao longo de um período relevante (dimensão temporal do estudo).

Especificamente para o caso dos materiais de construção, em que a ACV é conduzida do berço ao portão da indústria, define-se uma declaredunit, que deve ser medida em unidades de massa, área, comprimento, volume ou itens (BUILDING..., 2013). Isso porque o uso dessas métricas facilita a comparação de diferentes materiais de construção pelo projetista.

De acordo com a NBR ISO 14044 (ABNT, 2009), os requisitos de qualidade dos dados devem ser especificados para que o objetivo e o escopo da ACV possam ser alcançados. Os dados fornecidos pela indústria devem cobrir o período de 1 ano de produção, o qual deve estar documentado, e derivações desse intervalo devem ser justificadas (BUILDING..., 2013). Ainda, dados medidos devem ser atualizados a cada 5 anos, e dados estimados, a cada 10 anos.

\section{Análise de inventário (ICV)}

$\mathrm{Na}$ etapa de ICV, são computadas todas as entradas e saídas associadas a cada produto, incluindo matérias-primas, embalagem e itens de consumo.Em muitos casos, esse processo pode ser bastante exaustivo, dependendo do número de fluxos considerados nele. Nesse caso, costuma-se considerar um critério de corte para produtos com pouca participação no processo e/ou que geram baixo impacto ambiental. Porém, é importante garantir que efeitos ambientais significativos não sejam omitidos ao ignorar substâncias de baixo fluxo mássico. Para isso, certas convenções são normalmente aplicadas (BUILDING..., 2013). No caso de dados insuficientes ou falta de dados para determinada unidade de processo, o critério de corte comumente aplicado é $1 \%$ do fluxo mássico total de entradas do processo. O total de entradas desconsideradas por módulo deve ser inferior a $5 \%$ da energia usada e da massa de entrada. São exceções os casos em que esses processos tenham efeitos significativos ou elevado uso de energia em sua extração, uso ou disposição; ou quando sejam classificados como resíduos perigosos. Nestes casos, mesmo com baixa participação no processo, esses fluxos devem ser considerados.

Balanços de massa podem assegurar que as entradas consideradas são suficientes para produzir todas as saídas, incluindo a geração de resíduos. No geral, uma combinação de dados de literatura com outros obtidos diretamente com a indústria é preferível para o cálculo de declarações ambientais de produto, como segue:

10 Passuello, A. C. B.; Oliveira, A. F. de; Costa, E. B. da; Kirchheim, A. P. 
(a) dados medidos derivados de processos específicos devem ser usados na elaboração de um ACV. Esses dados também podem ser aplicados para processos relacionados a entradas da indústria do produto em estudo, quando disponíveis; e

(b) dados calculados e estimados podem ser usados para os demais processos que estão além do controle da indústria em estudo (i.e., produção de matéria-prima, instalação, uso e disposição do produto).

Uma vez coletados todos os dados necessários, precede-se à parte de cálculo do ICV, em que os dados coletados são ajustados às unidades de processo e à unidade funcional. $\mathrm{O}$ resultado dessa etapa é uma quantificação de todos os recursos utilizados e de todas as emissões associadas à produção de determinada quantidade (unidade funcional) do produto em estudo. Por ser de difícil entendimento para a população em geral, sugere-se a aplicação da AICV para melhor compreensão dos impactos relacionados ao sistema de produto em estudo.

\section{Avaliação de impacto do ciclo de vida}

Na avaliação de impacto do ciclo de vida (AICV), os dados coletados na etapa anterior (ICV) são aplicados considerando os conhecimentos gerados nos pontos anteriores. Estão disponíveis na literatura vários trabalhos relacionados a métodos de avaliação de impacto de ciclo de vida (BOVEA; GALLARDO, 2006). Diversas metodologias de AICV foram desenvolvidas em todo o mundo nas últimas décadas, entre as quais se destacam: CML (GUINÉE et al., 2000), EDIP 2003 (WENZEL; HAUSCHILD, 1997), Eco-Indicator (GOEDKOOP; SPRIENSMA, 2000) e IMPACT 2002+ (JOLLIET et al., 2003). Cada uma destas metodologias possui uma lista de categorias de impacto a ser avaliadas.

Existem muitos estudos sobre quais as categorias de impacto mais importantes para aplicação à construção civil. A norma inglesa EN 15804 (BUILDING..., 2013) aconselha o uso dos fatores de classificação do CML versão IA, que são: mudança climática, depleção do ozônio estratosférico, acidificação do solo e da água, eutrofização, ozônio fotoquímico e depleção de recursos abióticos (fóssil e não fóssil). Glass et al. (2013) afirmam que é importante considerar, além do consumo energético e geração de resíduos, também os indicadores recomendados pela norma inglesa. Bösch et al. (2007) indicam o uso de uma nova métrica chamada CExD (do inglês CumulativeExergyDemand). Dita métrica considera a "exergia" (BÖSCH et al., 2007) dos recursos utilizados no ciclo de vida em estudo. Essa ferramenta pode ser especialmente interessante para o caso da construção civil, por avaliar conjuntamente a demanda energética e de recursos de uma perspectiva da qualidade energética, integrando recursos energéticos e nãoenergéticos, tão amplamente aplicados na indústria da construção civil. Finalmente, o Conselho Brasileiro de Construção Sustentável (CBCS) indica cinco impactos prioritários para uma metodologia de ACV simplificado que seja viável à construção brasileira: mudanças climáticas, uso de recursos naturais, consumo de energia, geração de resíduos e consumo de água (AGOPYAN; JOHN, 2011).

\section{Interpretação do ciclo de vida}

Esta etapa inclui diversos elementos, tais como a identificação de questões significativas da análise, verificações de completeza, sensibilidade e consistência, conclusões, limitações e recomendações (ABNT, 2009), servindo de base para a tomada de decisão em relação à concepção e/ou uso do produto avaliado. No caso de ACV comparativa, essa fase confrontará os resultados dos distintos produtos avaliados, indicando possíveis diferenças em relação a seus impactos ambientais potenciais.

\section{Aplicação da ACV para avaliação ambiental da pegada de carbono de materiais cimentícios alternativos: estudo de caso}

A importância da seleção de materiais de construção no desempenho ambiental de edificações é destacada por vários autores (PASSER; KREINER; MAYDL, 2012). Dados do inventário brasileiro de emissões de gases efeito estufa comprovam que, no Brasil, as emissões desses gases para edificações durante a fase de produção e no transporte de materiais são mais importantes que os associados ao consumo de energia durante o uso do edifício (AGOPYAN; JOHN, 2011).Para efetuar esse tipo de análise, é necessário o acesso a bases de dados de ciclo de vida, bem como a coleta de dados na indústria produtora de materiais de construção, tal como descrito anteriormente. Porém, no caso de materiais de construção inovadores, faz-se necessário extrapolar dados de laboratório para a estimativa do possível impacto desses produtos, caso fossem produzidos em escala industrial. A seguir, propõe-se uma metodologia simplificada de aplicação da ACV à produção de um material 
cimentício inovador, o clínquersulfoaluminato de cálcio belítico (CSAB), produzido em escala laboratorial com o uso de resíduos.

O CSAB é considerado mais amigável ambientalmente que o convencional clínquer Portland, devido à redução da emissão de $\mathrm{CO}_{2}$ proporcionada pela baixa temperatura de queima (aproximadamente $200{ }^{\circ} \mathrm{C}$ menos que o Portland) e menor teor de calcário empregado em sua produção. Ainda, o clínquer resultante é mais friável (devido a sua elevada porosidade), reduzindo a energia necessária para a moagem. Além disso, o CSAB contém quantidades elevadas de enxofre, o que o torna excelente consumidor de materiais e/ou resíduos que contenham esse material, tais como resíduos de cinzas de leito fluidizado e resíduos de gesso. A utilização desses resíduos na produção de clínquer CSAB diminui a deposição em aterro, reduzindo, assim, o impacto ambiental da fabricação desse clínquer (CHEN, 2009; COSTA, 2013).

Esse cimento ainda está em fase de testes, não tendo sido utilizado em escala real. No entanto, vários pesquisadores desenvolvem estudos nessa linha, como Quillin (2001), Chen (2009), Jewellet al. (2009), Martín-Sedeñoet al. (2010), Senffet al. (2011), Álvarez-Pinazoet al.(2012) e Costa et al.(2013). Em âmbito industrial, a empresa Lafarge vem desenvolvendo e divulgando a produção de uma nova linha de cimentos CSAB há alguns anos, a qual patentearam sob o nome de Aether ${ }^{\circledR}$.No entanto, as pesquisas de desenvolvimento do produto ainda estão sendo realizadas, e a primeira produção industrial do produto foi divulgada recentemente $^{1}$. A empresa justifica que o projeto possibilitará a redução da emissão de $33 \%$ de $\mathrm{CO}_{2}$ por tonelada de cimento até 2020. Da mesma forma, a indústria cimenteira alemã Heidelberg Cementtambém desenvolve, desde 2009, estudos nessa linha através de pesquisadores do centro de tecnologia da indústria ${ }^{2}$ e aponta bons resultados com o primeiro teste de queima a ser realizado em uma das indústrias da cimenteira.

Para estimar a pegada de carbono do clínquer CSAB, propõe-se a aplicação da metodologia $\mathrm{ACV}$, tal como descrito na continuação.

\section{Definição de objetivo e escopo}

O objetivo do presente estudo é avaliar a pegada de carbono do clínquer CSAB produzido em laboratório, estimando qual seria o valor de sua pegada de carbono caso fosse fabricado em escala industrial no Brasil. A unidade funcional (ou declairedunit) do estudo é $1 \mathrm{~kg}$ de clínquer CSAB. Dados ambientais foram obtidos da base de dados ecoinvent v.3 (ECOINVENT..., 2014). O método de AICV empregado é o IPCC 2007 para um período de 100 anos, conforme indicado pela norma ISO 14067 (INTERNATIONAL..., 2013).

A Figura 1 mostra o sistema de produto em estudo e suas fronteiras. Estão considerados no escopo do estudo a produção de combustíveis, extração e produção de matérias-primas e materiais auxiliares, calcinação do clínquer em forno rotativo, consumo de eletricidade para produção do clínquer e de combustíveis, matérias-primas e materiais auxiliares, bem como o transporte de todos os materiais utilizados. Estão fora do escopo do estudo a moagem e a mistura do clínquer e embalagem do cimento ou seu transporte ao consumidor final, uma vez que o escopo da análise é do berço à porta da indústria.

\section{Análise de Inventário}

$\mathrm{Na}$ análise de inventário foram coletados dados medidos, estimados e calculados, conforme descrito no item Análise de inventário (ICV). Pelo fato de se tratar de um material de construção inovador, não foi possível a medição de dados diretamente na indústria. Por isso, primeiramente, realizou-se uma revisão bibliográfica de estudos de caso relacionados à pegada de carbono do clínquer Portland, dada a similaridade dos processos produtivos. Constatou-se que a maior parte das emissões de gases efeito estufa no CV do clínquer Portland é devida aos processos de calcinação e ao consumo energético do forno rotativo. Como muitos desses estudos foram desenvolvidos no exterior, considerou-se que também seria importante avaliar o consumo energético típico de uma indústria cimenteira brasileira (BAJAY, 2009) e a produção de eletricidade e combustíveis, considerando as peculiaridades da matriz energética do país.

Desse modo, foram selecionadas as medições que deveriam ser executadas em laboratório: emissão de $\mathrm{CO}_{2}$ no processo de calcinação; e consumo energético do forno rotativo.

${ }^{1}$ http://www.globalcement.com/news/item/1374-lafargeproduces-aether-clinker-for-first-time.

${ }^{2}$ http://www.heidelbergcement.com/global/en/company/press_ media/archive/press_releases_2013/2013-03-26.htm. 
Figura 1 - Sistema de produto para a produção de clínqueres

Fronteiras do sistema

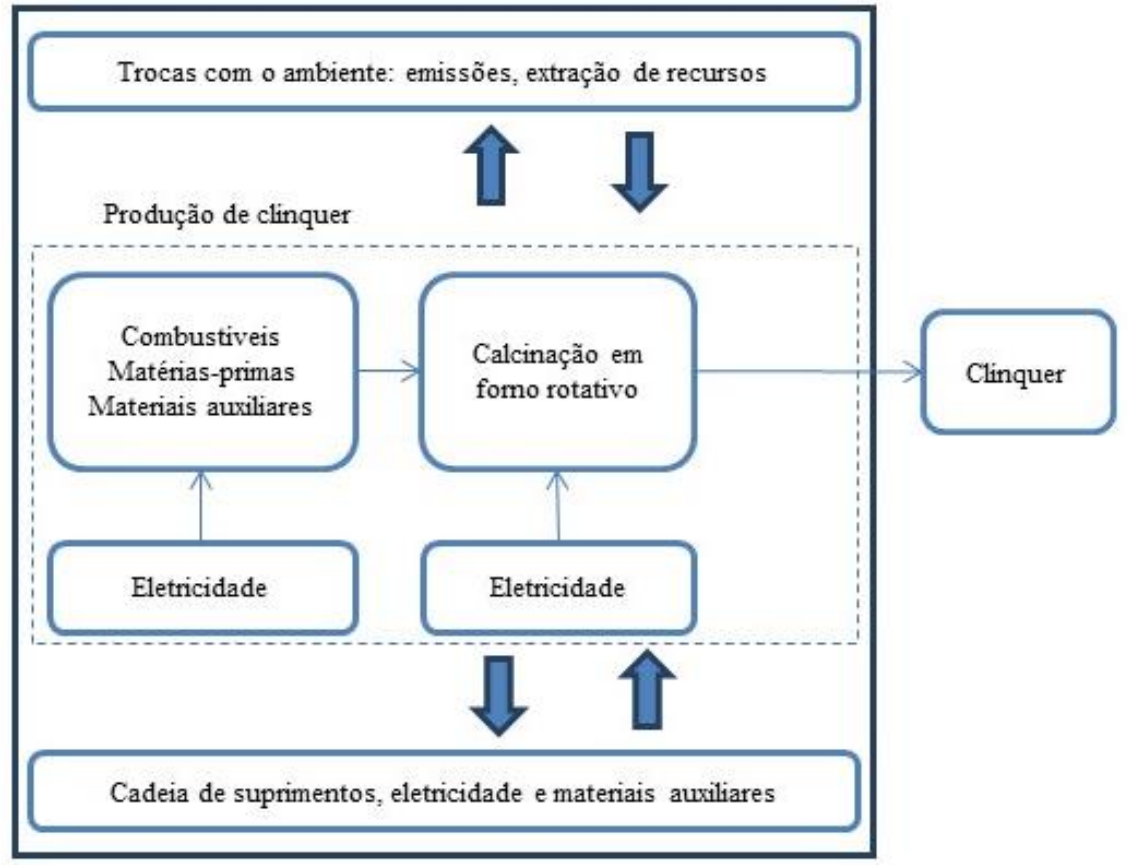

\section{Estimativa das emissões de $\mathrm{CO}_{2}$ no processo de calcinação}

As estimativas das emissões da farinha precursora para os clínqueres CSAB foram calculadas pelas Equações 1 e 2, obtidas a partir de cálculos estequiométricos em função dos teores de óxidos $\mathrm{SO}_{3}$ e $\mathrm{CaO}$ do clínquer. Nesse caso, consideram-se a calcita $\left(\mathrm{CaCO}_{3}\right)$ e o sulfato de cálcio $\left(\mathrm{CaSO}_{4}\right.$ ou $\mathrm{CaSO}_{4} \cdot \mathrm{xH}_{2} \mathrm{O}$ ) como fontes de $\mathrm{CaO}$ para sinterização das fases do clínquer CSAB. Para o clínquer Portland, foram usadas as Equações 3 e 4 (COSTA, 2013). A Tabela 1 apresenta a composição completa de óxidos dos clínqueres produzidos a partir das farinhas CSAB e Portland comparados neste trabalho, que serviram de base para os cálculos realizados.

$$
\begin{aligned}
& \mathrm{CO}_{2}{ }_{C S A B}\left(\frac{\mathrm{kg}}{\text { ton }}\right)=C t_{C S A B} *[100-\% \text { Adição }] * \\
& {\left[80 * \% \mathrm{CaO}-56 * \% \mathrm{SO}_{3}\right]} \\
& C t_{C S A B}=\left[\frac{44}{(56 * 80)} * \frac{10}{\left(100+\% \text { CaSO }_{4}\right)}\right] \quad \text { Eq. } 2 \\
& \mathrm{CO}_{2_{\text {Portland }}}\left(\frac{\mathrm{kg}}{\text { ton }}\right)=C t_{\text {Portland }} * \% \mathrm{CaO} *(100- \\
& \text { \%Adição) } \\
& \text { Eq. } 3 \\
& C_{\text {Portland }}=\left[\frac{44}{56} * \frac{10}{\left(100+\text { CaSO }_{4}\right)}\right] \quad \text { Eq. } 4
\end{aligned}
$$

Como as estimativas foram realizadas para a produção de clínqueres, não foi considerada a adição de gipsita, tendo sido ajustado seu valor para zero em todas as equações.

\section{Estimativa do consumo energético em forno rotativo}

A estimativa do consumo energético em forno rotativo foi feita a partir de análise termogravimétrica. A análise termogravimétrica permite a determinação analítica de variações de massa da amostra (ganho ou perda) durante seu aquecimento no interior de um forno sob aquecimento contínuo e uniforme. As variações de massa podem ser causadas por alterações físicas no material, tais como sublimação, evaporação e sorção, ou por interações químicas (SILVA, 2001).

Os testes foram realizados em um equipamento Mettler Toledo TGA/TSO SDTA 851e. As amostras das farinhas precursoras dos clínqueres foram colocadas em porta-amostra de platina. A temperatura máxima de análise das farinhas foi de $1.000^{\circ} \mathrm{C}$.

Tabela 1- Composição de óxidos dos clínqueres CSAB e Portland 


\begin{tabular}{l|c|c|c|c|c|c|c|c|c}
\hline & $\mathbf{C a O}$ & $\mathrm{Al}_{2} \mathbf{O}_{3}$ & $\mathrm{SiO}_{2}$ & $\mathrm{SO}_{3}$ & $\mathrm{Fe}_{2} \mathbf{O}_{3}$ & $\mathbf{M g O}$ & $\begin{array}{c}\text { Outras } \\
\text { impurezas }\end{array}$ & $\begin{array}{c}\text { Perda } \\
\text { ao fogo }\end{array}$ & Total \\
\hline $\begin{array}{l}\text { Portland } \\
\text { (MARINGOLO, }\end{array}$ & 67,7 & 3,9 & 18,4 & - & 3,2 & 6,7 & - & - & 100 \\
$\begin{array}{l}\text { 2001) } \\
\text { CSAB (COSTA, }\end{array}$ & 48,8 & 16,9 & 9,1 & 12,4 & 3,73 & 0,07 & 0,74 & 0,56 & 100 \\
2013)
\end{tabular}

Nota: ${ }^{1}$ As análises químicas de elementos maiores e menores em porcentagem de óxidos foram efetuadas com espectrômetro de fluorescência de raios X Ragaku, modelo RIX 200, com tubo Rh (MARINGOLO, 2001). ${ }^{2}$ Os teores apresentados foram determinados por análise semiquantitativa sem padrões (standardless) com análise de elementos químicos de flúor a urânio, em espectrômetro por fluorescência de raios X Axios Advanced, marca PANalytical (COSTA, 2013).

A partir dos resultados das análises, é possível estimar a perda de massa (PM, em \%) na fabricação dos clínqueres Portlande CSAB, produzidos em laboratório a partir de reagentes puros tal como descrito por Costa et al. (2013) e Costa (2013) respectivamente. Considerando simplificadamente que uma menor perda de massa pode ser associada a um menor consumo energético, uma vez que para a mesma quantidade de energia produz-se mais material, é possível estimar a demanda energética (DE, em $\mathrm{MJ} \mathrm{kg}^{-1} \mathrm{de}$ clínquer) da produção do clínquer CSAB em comparação ao Portland (Eq. 5).

$D E_{C S A B}=D E_{\text {Portland }}\left(\frac{100-P M_{\text {Portland }}}{100-P M_{C S A B}}\right)$

Eq. 5

É importante salientar que esta é uma estimativa bastante conservadora, pois não leva em conta que o clínquer CSAB é produzido a temperaturas mais baixas que o Portland $\left(1.250^{\circ} \mathrm{C}\right.$ contra $\left.1.450^{\circ} \mathrm{C}\right)$, porém pode servir de base para uma primeira aproximação, tratando-se das estimativas mais precisas possíveis em um experimento em escala piloto.

\section{Resultados}

\section{Estimativa das emissões de $\mathrm{CO}_{2}$ durante o processo de calcinação}

A partir da aplicação dos dados de produção dos clínqueres em laboratório (Tabela 2) às equações acima descritas, foi possível estimar as emissões de $\mathrm{CO}_{2}$ relacionadas à calcinação da farinha, considerando que a emissão de $\mathrm{CO}_{2}$ nos fornos de cimento está diretamente relacionada à decomposição do calcário $\left(\mathrm{CaCO}_{3} \rightarrow \mathrm{CaO}+\mathrm{CO}_{2}\right.$ (Eq. 5)). O teor de $\mathrm{CaO}$ considerado no clínquer Portland foi de $67,7 \%$, de acordo com os estudos de Maringolo (2001),resultando em uma emissão de $532 \mathrm{~kg}$ de $\mathrm{CO}_{2}$ por tonelada de material produzido. Também foi possível constatar, a partir de ensaios de fluorescência de raios $\mathrm{X}$ (FRX), que o clínquer $\mathrm{CSAB}$ possui cerca de $48,8 \%$ de $\mathrm{CaO}$ e de $9,10 \%$ de $\mathrm{SO}_{3}$ (COSTA, 2013). Pela aplicação das Equações (1) e (2), temos que, para uma tonelada de material, há emissão de $330 \mathrm{~kg}$ de $\mathrm{CO}_{2}$, uma redução de $36 \%$ na pegada de carbono em comparação ao clínquer Portland para o processo de clinquerização.

\section{Estimativa do consumo energético em forno rotativo}

Os resultados da análise termogravimétrica, como mostrados no gráfico da Figura 2 e Tabela 3 , indicam que há menor perda de massa $(\mathrm{PM})$ da farinha precursora do clínquer CSAB em relação à farinha precursorado clínquer Portland (30,32\% contra 36,81\%). A Tabela 1 apresentou a composição de óxidos dos respectivos clínqueres produzidos a partir das farinhas. Dessa forma, a produção do CSAB necessita 9,32\% menos energia (Eq. 5). Considerou-se, pois, a redução dessa demanda energética para o aquecimento direto do forno. Outros consumos energéticos da indústria, tais como para iluminação e calor de processo, foram mantidos constantes na análise. Cabe destacar, também, que a redução no consumo energético é bastante significativa, uma vez que o aquecimento direto corresponde a 79,3\% do consumo energético da indústria cimenteira no Brasil (BAJAY, 2009).Vale ressaltar novamente que esta é uma estimativa bastante conservadora, visto que não considera o consumo de combustível diferenciado devido aos patamares de queima diferentes. 
Tabela 2- Dados de cálculo para estimativa de emissão de gases efeito estufa (em $\mathrm{kg} \mathrm{CO}_{2} \mathrm{eq}$ ) durante a etapa de clinquerização, para os dois clínqueres produzidos em laboratório

\begin{tabular}{c|c|c|c|c|c|c}
\hline & $\begin{array}{c}\mathrm{CaSO}_{\mathbf{4}} \\
(\boldsymbol{\%})\end{array}$ & $\mathbf{C t}$ & $\begin{array}{c}\mathbf{C a O} \\
(\boldsymbol{\%})\end{array}$ & $\begin{array}{c}\mathbf{S O}_{3} \\
(\boldsymbol{\%})\end{array}$ & $\begin{array}{c}\text { Adição } \\
(\boldsymbol{\%})\end{array}$ & $\begin{array}{c}\text { Emissões CO } \\
(\mathbf{k g} / \mathbf{k g} \text { clínquer })\end{array}$ \\
\hline Portland & 0 & $7,86 \cdot 10^{-2}$ & 67,70 & - & 0 & 0,53 \\
CSAB & 0 & $9,82 \cdot 10^{-4}$ & 48,80 & 9,10 & 0 & 0,33 \\
\hline
\end{tabular}

Figura 2 - Curvas da análise termogravimétrica das farinhas de clínquer Portland e CSAB

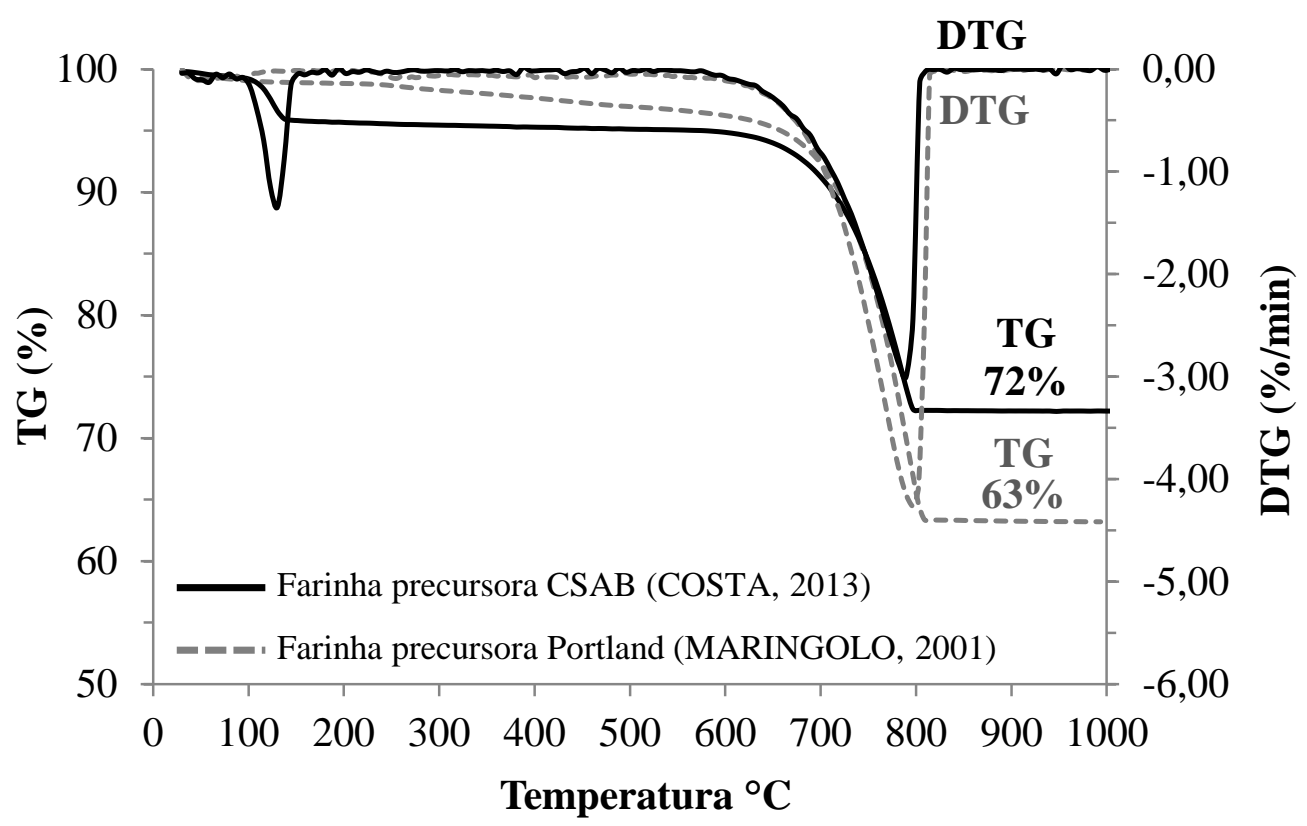

Tabela 3- Dados para a estimativa do consumo energético durante a clinquerização do CSAB

\begin{tabular}{l|c|c}
\hline & PM(\%) & Consumo energético(MJ/kg clínquer) \\
\hline Portland & 36,81 & $3,55^{1}$ \\
CSAB & 30,32 & 3,21 \\
\hline
\end{tabular}

Nota: ${ }^{1}$ Bajay (2009).

\section{ACV comparativa dos clínqueres avaliados}

Em paralelo aos estudos realizados em laboratório, foi desenvolvida a ACV do clínquer Portland brasileiro, a partir de estimativas da indústria nacional (BAJAY, 2009) e bases de dados consagradas internacionalmente (ECOINVENT..., 2014). A partir dessa análise, foi possível estimar a pegada de carbono do clínquer Portland produzido no Brasil. A seguir, os dados comparativos para os clínqueres CSAB e Portland obtidos em laboratório (Tabelas 2 e 3 ) foram aplicados para se estimar a pegada de carbono do clínquer CSAB, extrapolando os dados obtidos para o clínquer Portland.

Os resultados da pegada de carbono dos clínqueres avaliados estão contemplados na Tabela 4. Enquanto o clínquer Portland produzido no Brasil apresenta uma pegada de carbono de 0,99 kg
$\mathrm{CO}_{2}$ eq $\mathrm{kg}^{-1}$ clínquer, o $\mathrm{CSAB}$ apresenta uma pegada de $0,75 \mathrm{~kg} \mathrm{CO}_{2} \mathrm{eq} \mathrm{kg}^{-1}$ clínquer, o que representa uma redução de $22 \%$ nas emissões de gases do efeito estufa. Essa redução se deve especialmente às reações da etapa de calcinação (Equações de 1 a 4). Os valores encontrados para a pegada de carbono do clínquer Portland são similares aos apresentados em outros estudos, tais como os apresentados por Valderrama et al. (2011) (entre 0,94 e $0,99 \mathrm{~kg} \mathrm{CO} \mathrm{kg}^{-1}$ clínquer), em um estudo de uma cimenteira espanhola que opera uma nova linha de produção com tecnologia de última geração. Em contraste, os resultados obtidos neste estudo são inferiores aos apresentados por Bösch, Koehler e Hellweg (2009) $\left(1,14 \quad \mathrm{~kg} \quad \mathrm{CO}_{2} \quad \mathrm{~kg}^{-1}\right.$ clínquer) para clínqueres produzidos em fornos pela via seca, similar ao que é aplicado no Brasil atualmente. Cabe destacar, ainda, que os dados do Conselho Mundial para o 
Desenvolvimento Sustentável (CEMENT..., 2011) são um pouco inferiores aos obtidos neste estudo (0,87 $\mathrm{kg} \mathrm{CO}_{2} /$ ton de clínquer, para o ano 2006). Porém, os dados do CSI consideram apenas as emissões associadas ao processo de calcinação e queima, sem considerar as emissões relacionadas a outras etapas do ciclo de vida (tais como produção de eletricidade). As diferenças entre os resultados dos diferentes estudos se devem basicamente aos pressupostos e limitações das análises, tais como características operacionais das indústrias, consumo de diferentes combustíveis e matriz energética dos locais de estudo.

Considerando-se a produção do clínquer Portland no Brasil como cenário-base para a comparação, igualando sua pegada de carbono a 100\% (Figura 3 ), pode-se contemplar as contribuições de cada uma das unidades de processo na pegada de carbono dos produtos avaliados, bem como as diferenças observadas para os dois produtos avaliados.

\section{Discussão}

Mediante a avaliação simplificada do ciclo de vida dos clínqueres produzidos em laboratório, é possível constatar redução significativa nas emissões de gases efeito estufa através da produção de um clínquer alternativo (CSAB). É sabido que a produção de cimentos com adições também colabora na redução de emissões de gases efeito estufa desse material. Porém, pelo fato de o CSAB ser mais friável, sua moagem demandaria menos energia, o que também resultariaem uma melhoria em sua pegada de carbono, já que a moagem é o processo que mais demanda eletricidade na planta (VALDERRAMAet al., 2011). Nesse sentido, cabem mais estudos de ACV de materiais cimentícios para avaliar quais as alternativas mais vantajosas no uso desse material.

Tabela 4 - Resultados da pegada de carbono dos clínqueres avaliados ( $\mathrm{kg} \mathrm{CO}_{2}$ eq $\mathbf{~ k g}^{-1}$ clínquer)

\begin{tabular}{l|c|c|c}
\hline & Clínquer CSAB & Clínquer Portland & Redução CSAB/Portland \\
\hline Calcinação & 0,33 & 0,53 & $37 \%$ \\
Queima de combustíveis fósseis & 0,30 & 0,33 & $9 \%$ \\
Produção de combustíveis fósseis & $6,80 \cdot 10^{-2}$ & $7,28 \cdot 10^{-2}$ & $7 \%$ \\
Resto CV & $5,33 \cdot 10^{-2}$ & $5,33 \cdot 10^{-2}$ & $0 \%$ \\
Total & 0,75 & 0,99 & $24 \%$ \\
\hline
\end{tabular}

Figura 3 - Comparação dos cenários para as categorias de caracterização relacionadas à pegada de carbono



16 Passuello, A. C. B.; Oliveira, A. F. de; Costa, E. B. da; Kirchheim, A. P. 
Cabe salientar que esta é uma estimativa bastante simplificada e conservadora, e experimentos em escala piloto são necessários para validar estes resultados. Porém, os resultados demonstram o potencial da ACV para avaliar o impacto ambiental da produção de materiais de construção inovadores em escala laboratorial.

Tal como se observa na Figura 3 e Tabela 4, as etapas do ciclo de vida do clínquer que possuem as maiores emissões de gases efeito estufa são a calcinação, a queima e a produção de combustíveis fósseis. A fabricação de clínqueres de reduzido impacto ambiental do tipo CSAB apresentado no presente estudo totalizaria uma redução de $24 \%$ das emissões relacionadas a essas unidades de processo, através da redução de $37 \%$ das emissões relativas à calcinação, $9 \%$ relacionadas à queima $\mathrm{e}$ $7 \%$ à produção de combustíveis fósseisquando comparados a clínqueres Portland convencionais.

Uma das principais dificuldades da aplicação desse método é a escassez de dados de ICV de materiais de construção produzidos no Brasil, sendo a maioria deles associada a materiais cerâmicos (HADDADet al., 2013; GOMES; SALGADO; HOTZA,2012). A falta de dados nacionais é uma importante limitação para a aplicação da ACV no Brasil, mas o uso de dados internacionais é válido, contanto que se encontrem dados fiáveis e baseados em processo tecnológico similar. Também é importante salientar que, no caso da estimativa de pegada de carbono, um dos dados mais relevantes a ser avaliado é o consumo energético. A existência de dados de consumos energéticos do setore da matriz energética nacional permite uma correta avaliação de sua pegada de carbono. Sem embargo, é essencial que centros de pesquisa e indústrias realizem parcerias para desenvolver bases de dados de ciclo de vida de produtos da construção civil, o que permitirá que futuramente projetistas façam escolhas mais conscientes em relação à seleção de materiais de construção, tal como sugere a NBR 15575 (ABNT, 2013).

Outra importante limitação relacionada à avaliação ambiental de materiais de construção inovadores é a inexistência de produção do material em escala real. Nesse caso, a ACV provê as bases para a comparação de materiais em escala laboratorial, servindo de guia para a estimativa dos possíveis impactos da produção deles em escala industrial.Além disso, essa metodologiaé internacionalmente aplicada para a confecção de declarações ambientais de produto, ferramenta que permite ao fornecedor/fabricante declarar as emissões associadas ao ciclo de vida de seu produto, da mesma forma com que declara suas propriedades técnicas.
Uma importante limitação da aplicação da ACV a materiais de construção está relacionada a como avaliar a durabilidade do material em comparação a seu desempenho ambiental. A durabilidade dos produtos influencia decisivamente o período em que a construção vai prestar serviços e a quantidade de recursos na manutenção. Por isso, considera-se que está relacionada ao impacto ambiental, social e econômico da edificação. Porém, muitos pesquisadores do tema Green Buildings têm surpreendentemente negligenciado esse fato, o que pode ser relacionado a que a ACV seja normalmente aplicada para avaliar produtos que possuem ciclo de vida curto se comparados a uma edificação, cuja vida útil de projeto pode ser de mais de 100 anos. No caso dos clínqueres e do cimento, esse fato dependerá não somente das características do produto como dos critérios de projeto. De posse dessa informação e do perfil ambiental do produto, o projetista certamente disporá de informações relevantes para realizar uma tomada de decisão mais consciente, considerando critérios sociais, econômicos e ambientais.

\section{Considerações finais}

Internacionalmente aplicada para a avaliação ambiental de produtos, processos e/ou serviços, a ACV é uma metodologia considerada apropriada para a avaliação ambiental de materiais de construção inovadores, sempre que aplicada de forma transparente e com metodologia consistente com a normativa vigente.Dita metodologia já é empregada no desenvolvimento de declarações ambientais de produtos da construção civil e indicada para a avaliação da pegada de carbono de produtos, conforme a normativa vigente (INTERNATIONAL..., 2013). Sua aplicabilidade fica comprovada através do estudo de caso comparativo de clínqueres produzidos em laboratório, em que se demonstrou a redução da pegada de carbono de clínqueres alternativos do tipo CSAB em comparação ao clínquer Portland. Porém, para uma correta avaliação das vantagens ambientais de um material de construção inovador em comparação a um insumo tradicionalmente aplicado na indústria da construção civil, faz-se necessário também avaliar a vida útil de serviço dos materiais estudados. A integração dos conceitos de vida útil e impacto ambiental é atualmente um desafio, e mais estudos são necessários para agregar esses dados de maneira transparente, o que facilitará sua aplicação por projetistas e permitirá tomadas de decisão mais conscientes com vistas à sustentabilidade. 


\section{Referências}

ABEYSUNDRA, U. G. Y. et al.Environmental, Economic and Social Analysis of Materials For Doors and Windows in Sri Lanka. Building and Environment, v. 42, n. 5, p. 2141-2149, 2007.

ÁLVAREZ-PINAZO, G. et al.Rietveld Quantitative Phase Analysis of YeelimiteContaining Cements. Cement and Concrete Research, v. 42, n. 7, p. 960-971, 2012.

ASSOCIAÇÃO BRASILEIRA DE NORMAS TÉCNICAS. NBR ISO 14040: gestão ambiental: avaliação do ciclo de vida: princípios e estrutura. Rio de Janeiro, 2009.

\section{ASSOCIAÇÃO BRASILEIRA DE NORMAS} TÉCNICAS. NBR 15575-1: edificações habitacionais: desempenho: parte 1: requisitos gerais. Rio de Janeiro, 2013.

AGOPYAN, V.; JOHN, V. O Desafio da Sustentabilidade na Construção Civil. São Paulo: Blucher, 2011.

BAJAY, S. V. Relatório Setorial: setor cimenteiro. In: DORILEO, I. L. D. (Coord.). Oportunidades de Eficiência Energética Para Indústria. Brasília: CNI, 2009.

BISWAS, W. K. Carbon Footprint and Embodied Energy Assessment of a Civil Works Program in a Residential Estate of Western Australia.The International Journal of Life Cycle Assessment, v. 19, n. 4, p. 732-744, apr.2013.

BÖSCH, M. E.; KOEHLER, A.; HELLWEG, S. Model ForCradle-to-Gate Life Cycle Assessment of Clinker Production. Environmental Science and Technology, v. 43, n. 19, p. 7578-7583, 2009.

BÖSCH, M. E. et al. Applying Cumulative ExergyDemand (CExD) Indicators to the EcoinventDatabase. The International Journal of Life Cycle Assessment, v. 12, n. 3, p. 181-190, 2007.

BOSOAGA, A.; MASEK, O; OAKEY, J. E. $\mathrm{CO}_{2}$ Capture Technologies ForCement Industry. Energy Procedia, v. 1, n. 1, p. 133-140, 2009.

BOVEA, M. D.; GALLARDO, A. The Influence of Impact Assessment Methods on Materials Selection For eco-Design. Materials \& Design, v. 27, n. 3, p. 209-215, 2006.
BRASIL. MINISTÉRIO DO

DESENVOLVIMENTO, INDÚSTRIA E

COMÉRCIO EXTERIOR. CONSELHO

NACIONAL DE METROLOGIA, N. Resolução

n $^{\circ} 01$, de 6 de abril de 2011, que dispõe sobre a

Aprovação do Regimento Interno e da composição do Comitê Gestor do Programa Brasileiro de Avaliação do Ciclo de Vida e dá outras providências. Brasília: 2011.

\section{BUILDING RESEARCH}

ESTABLISHMENT.Product Category Rules for Type III Environmental Product Declaration of Construction Products to EN 15804:2012. Watford: BRE, 2013.

CEMENT SUSTAINABILITY INITIATIVE.CO and Energy Accounting and Reporting Standard for the Cement Industry: the Cement $\mathrm{CO}_{2}$ and Energy Protocol. Version 3.0.Washington:World Business Council for Sustainable Development, 2011.

CHEN, I. A. Synthesis of Portland Cement and Calcium Sulfoaluminate-BeliteementFor Sustainable Development and Performance. Austin: The University of Texas, 2009.

COSTA, E. B. Aproveitamento do Resíduo de Anodização do Alumínio na Produção do Cimento Sulfoaluminato de Cálcio Belítico. Porto Alegre, 2013. Dissertação (Mestrado em Engenharia Civil) - Escola de Engenharia, Universidade Federal do Rio Grande do Sul, Porto Alegre, 2013.

COSTA, E. B. et al.Clínquer Portland Com Reduzido Impacto Ambiental. Ambiente Construído, Porto Alegre, v. 13, n. 2, p. 75-86, abr./jun. 2013.

ECOINVENT CENTRE. EcoinventDatabase. Swiss Centre for Life Cycle Inventories. Disponível em: <www.ecoinvent.ch〉. Acesso em: 10 jan. 2014.

GLASS, J. et al.Future use of Life-Cycle Assessment in Civil Engineering.Proceedings ICE Construction Materials, v. 166, n. 4, p. 204212, 2013.

GOEDKOOP, M.; SPRIENSMA, L. Eco-Indicator 99: manual for designers, a damage oriented method for life cycle impact assessment. Methodology Report, 2000.

GOMES, J. M.; SALGADO, A. L. F.; HOTZA, D. Life Cycle Assessment of Ceramic Bricks.Materials Science Forum, v. 727/728, p. 815-820, 2012. 
GUINÉE, J. B. et al. Environmental Life Cycle Assessment: backgrounds. Leidei: MultiCopy, 2000.

HADDAD, A. N. et al. Life Cycle Assessment: a comparison of ceramic brick inventories to subsidize the development of databases in Brazil. Applied Mechanics and Materials, v. 431, p. 370-377, 2013.

HUIJBREGTS, M. A. J. et al. Evaluating Uncertainty in Environmental Life-Cycle Assessment: a case study comparing two insulation options for a Dutch one-family dwelling. Environmental Science \& Technology, v. 37, n. 11, p. 2600-2608, 2003.

HUNTZINGER, D. N.; EATMON, T. D. A lifeCycle Assessment of Portland CementManufacturing: comparing the traditional process with alternative technologies. Journal of Cleaner Production, v. 17, n. 7, p. 668-675, 2009.

INTERGOVERNMENTAL PANEL ON CLIMATE CHANGE.Climate Change 2014: mitigation of climate change. Cambridge: Cambridge Press, 2014.

INTERNATIONAL ORGANIZATION FOR STANDARDIZATION.ISO/TS 14067: carbon footprint of products: requirements and guidelines for quantification and communication. Geneva, 2013.

JEWELL, R. B. et al. Fabrication and Testing of CSAB Cements in Mortar and Concrete that Utilize Circulating Fluidized Bed Combustion Byproducts. In: WORLD OF COAL ASH (WOCA) CONFERENCE. Proceedings... Lexington, 2009.

JOLLIET, O. et al. IMPACT 2002+: a new life cycle impact assessment methodology.

International Journal of Life Cycle Assessment, v. 8 , n. 6 , p. 324-330, 2003.

JÖNSSON, ̊.; TILLMAN, A.-M.; SVENSSON, T. Life Cycle assessment of Flooring Materials: case study. Building and Environment, v. 32, n. 3, p. 245-255, 1997.

KORONEOS, C.; DOMPROS, A. Environmental Essessment of Brick Production in

Greece.Building and Environment, v. 42, n. 5, p. 2114-2123, 2007.

LUGT, P. et al. An Environmental, Economic and Practical Assessment of Bamboo as a Building Material ForSupporting Structures. Construction and Building Materials, v. 20, n. 9, p. 648-656, 2006.
MARINGOLO, V. Clínquer co-Processado: produto de tecnologia integrada para a sustentabilidade e competitividade da indústria de cimento. São Paulo, 2001. Tese (Doutorado em Mineralogia Aplicada) - Departamento de Mineralogia e Petrologia, Universidade de São Paulo, São Paulo, 2001.

MARTÍN-SEDEÑO, M. C. et al.Aluminum-Rich Belite Sulfoaluminate Cements: clinkering and early age hydration. Cement and Concrete Research, v. 40, n. 3, p. 359-369, 2010.

MEHTA, P. K.; MONTEIRO, P. J. M. Concreto: microestrutura, propriedades e materiais. 3. ed. São Paulo: Ibracon, 2008.

NEBEL, B.; ZIMMER, B.; WEGENER, G. Life Cycle assessment of Wood Floor Coverings: a representative study for the German flooring industry. International Journal of Life Cycle Assessment, v. 11, n. 3, p. 172-182, 2006.

NICOLETTI, G. M.; NOTARNICOLA, B.; TASSIELLI, G. Comparative Life Cycle Assessment of flooring materials: ceramic versus marble tiles. Journal of Cleaner Production, v. 10, n. 3, p. 283-296, 2002.

NORRIS, G. A.; YOST, P. A Transparent, Interactive Software Environment ForCommunicating Life-Cycle Assessment Results: an application to residential windows. Journal of Industrial Ecology, v. 5, n. 4, p. 1528, 2001.

OCHOA, L.; HENDRICKSON, C.; MATTHEWS, H. S. Economic Input-Output Life-Cycle Assessment of U.S. Residential Buildings.Journal of Infrastructure Systems, v. 8, n. 4, p. 132-138, 2002.

ORTIZ, O.; CASTELLS, F.; SONNEMANN, G. Sustainability in the Construction Industry: a review of recent developments based on LCA. Construction and Building Materials, v. 23, n. 1, p. 28-39, 2009.

PASSER, A.; KREINER, H.; MAYDL, P. Assessment of the Environmental Performance of Buildings: a critical evaluation of the influence of technical building equipment on residential buildings. The International Journal of Life Cycle Assessment, v. 17, n. 9, p. 1116-1130, 2012.

QUILLIN, K. Performance of Belitesulfoaluminate Cements. Cement and Concrete Research, v. 31, n. 9, p. 1341-1349, 2001.

SENFF, L. et al. Formulations of Sulfobelite cement Through Design of Experiments. Construction and Building Materials, v. 25, n. 8, p. 3410-3416, 2011. 
SILVA, D. A. D. Efeito dos Polímeros HEC e EVA na Microestrutura de Pastas de Cimento Portland. Florianópolis, 2001. Tese (Doutorado em Ciência e Engenharia de Materiais) - Escola de engenharia, Universidade Federal de Santa Catarina, Florianópolis, 2001.

VALDERRAMA, C. et al. Implementation of Best Available Techniques in Cement Manufacturing: a life-cycle assessment study. Journal of Cleaner Production, v. 25, n. 3, p. 60-67, 2011.

WENZEL, H.; HAUSCHILD, M. A. L.

Methodology, Tools and Case Studies in Product Development: environmental assessment of products. London: Chapman, 1997.

\section{Agradecimentos}

Os autores agradecem o aporte financeiro do Conselho Nacional de Desenvolvimento Científico e Tecnológico (CNPq), à Coordenação de Aperfeiçoamento de Pessoal de Nível Superior (Capes) e à Fundação de Amparo à Pesquisa do Estado do Rio Grande do Sul (Fapergs), através dos editais Universal 2012, DOCFIX e DTI 2013. Os autores agradecem aos revisores, cujos comentários permitiram a melhoria do trabalho apresentado.

Ana Carolina Badalotti Passuello

Departamento de Engenharia Civil, Escola de Engenharia | Universidade Federal do Rio Grande do Sul | Av. Osvaldo Aranha, $99,3^{\circ}$ andar, Centro | Porto Alegre - RS - Brasil | CEP 90035-190 | Tel.: (51) 3308-3353 | E-mail: anapassuello@gmail.com

Alexandre Führ de Oliveira

Departamento de Engenharia Civil, Escola de Engenharia | Universidade Federal do Rio Grande do Sul | Tel.: (51) 3308-3518 | E-mail: alexandre.fuhr@live.com

Eugênio Bastos da Costa

Departamento de Engenharia Civil, Escola de Engenharia | Universidade Federal do Rio Grande do Sul | Tel.: (51) 3308 -3518 | E-mail: ebc.eng@hotmail.com

\section{Ana Paula Kirchheim}

Departamento de Engenharia Civil, Escola de Engenharia | Universidade Federal do Rio Grande do Sul | Tel.: (51) 3308-3879 |

E-mail: anapaula.k@ufrgs.br

Revista Ambiente Construído

Associação Nacional de Tecnologia do Ambiente Construído

Av. Osvaldo Aranha, $99-3^{\circ}$ andar, Centro

Porto Alegre - RS - Brasil

CEP $90035-190$

Telefone: +55 (51) 3308-4084

Fax: +55 (51) 3308-4054

www.seer.ufrgs.br/ambienteconstruido

E-mail: ambienteconstruido@ufrgs.br

20 Passuello, A. C. B.; Oliveira, A. F. de; Costa, E. B. da; Kirchheim, A. P. 